\title{
Logos como distanciación intelectiva en Xavier Zubiri
}

José Alfonso Villa Sánchez

Recibido: 4 de mayo de 2019 • Aprobado: 30 de septiembre de 2019

\section{Resumen}

La investigación sobre la intelección en la obra de Zubiri tiene tres grandes partes: la aprehensión de la realidad, el logos sentiente y la razón sentiente. Este estudio se ubica en la segunda parte, la relativa al logos sentiente. La tesis de Zubiri es que el logos tiene una estructura dinámica, conformada por dos fases. En la primera, llamada fase de impelencia, el logos se distancia de lo meramente real hacia lo que lo real es en realidad. Este movimiento de impelencia es una distanciación intelectiva, cuyos momentos son la retracción intelectiva y la simple aprehensión en sus modos de percepto, ficto y concepto. Este trabajo se interna en esos dos momentos de la impelencia, pero antes de hacerlo da cuenta de unos antecedentes conceptuales que ayuden a entender la naturaleza del logos como distanciación. La segunda fase del movimiento del logos, donde llega a su plenitud, es la afirmación, tema que queda fuera por lo pronto de estas páginas.

Palabras clave: intelección, logos, realidad, Zubiri.

* Artículo de reflexión en relación con el problema de la inteligencia sentiente y el logos en Xavier Zubiri. Citar como: Villa, J. (2020). Logos como distanciación intelectiva en Xavier Zubiri. Análisis, 52(96), 183-202. Dol: https://doi. org/10.15332/21459169/5122

** Doctor en Filosofía por la Universidad Iberoamericana de la Ciudad de México, Profesor Investigador en el Instituto de Investigaciones Filosóficas de la Universidad Michoacana de San Nicolás de Hidalgo (Morelia, México). Miembro del Sistema Nacional de Investigadores del Consejo Nacional de Ciencia y Tecnología de México; miembro del Círculo Latinoamericano de Fenomenología. Líneas de investigación: fenomenología, hermenéutica y ontología.

Correo: alphonsovilla@hotmail.com. Dirección postal: Cerrada Catedral de Henares \#27, Misión San Diego, Morelia, Michoacán, México. C. P. 58095. ORCID: https://orcid.org/0000-0002-3254-0613 


\section{Logos as intellectual distancing in Xavier Zubiri}

José Alfonso Villa Sánchez

\section{A bstract}

Research on intellection in Zubiri's work has three major parts: the apprehension of reality, the sentient logos and the sentient reason. This study is set in the second part, the one related to the sentient logos. Zubiri's thesis is that the logos has a dynamic structure, consisting of two phases. In the first, called the impedance phase, the logos distances itself from the merely real towards what the real is in reality. This impedance movement is an intellectual distancing, whose moments are intellectual retraction and simple apprehension in their modes of perception, fiction and concept. This work is internalized in those two moments of the impedance, but before doing so, it gives account of some conceptual backgrounds that help to understand the nature of the logos as distancing. The second phase of the logos movement, where it reaches its fullness, is the affirmation, a topic that is left out of these pages for the time being.

Keywords: intellection, logos, reality, Zubiri. 


\section{Le logos en tant que distanciation intellective}

José Alfonso Villa Sánchez

\section{Résumé}

La recherche sur l'intellection chez Zubiri compte trois grandes parties: la appréhension de la realité, el logos «sentiente » et la raison «sentiente ». Cet article se situe dans la seconde partie, relative au « logos sentiente ». La thèse de Zubiri consiste à dire que le logos a une structure dynamique formée de deux phases. Dans la première, que l'on appelle $d^{\prime}$ " impelencia », le logos prend distance du purement réel vers ce qui le réel est en réalité. Ce mouvement crée une distanciation intellective dont les moments sont la rétraction intellective et la simple appréhension dans ses modes de percept, ficto et concept. Ce travail revient sur deux moments $\mathrm{d}^{\text {' }}$ " impelencia » tout en rappelant des concepts précédents afin de comprendre la nature du logos en tant que distanciation. La seconde phase du mouvement du logos, et qui constitue son accomplissement, c'est l'affirmation, sujet qui ne peut pas être développée dans cet article.

Mots clés : intellection, logos, réalité, Zubiri. 


\section{Introducción}

La siguiente reflexión es un análisis cuidadoso de ese primer momento del logos que Zubiri llama distanciación, bajo la conjetura de que este concepto de logos está a la raíz de la tesis de Heidegger, que entiende el logos como $\delta \eta \lambda$ oũv, como "hacer patente aquello de lo que se habla en el decir" (cf. Heidegger, 2003, p. 55). El momento de la patencia tiene su condición de posibilidad en el logos sentiente en tanto distanciación, según quedará evidenciado.

Si vamos directamente al asunto que entiende el $\operatorname{logos}^{1}$ como distanciación intelectiva, hay que decir dos cosas. En primer lugar, el logos intelige lo que lo real es en realidad; va de lo real a lo que eso, que ya es real en la intelección, es en realidad: recorre la distancia que hay entre la mera actualización de lo real en la intelección y lo que esa realidad es en realidad, también en la intelección (cf. Pintor-Ramos, 2006). El logos no es, pues, la patentización del ser del ente, como pensaba Heidegger; pero tampoco es un juicio en el que se relacionan ideas que han hecho abstracciones a partir de los datos proporcionados por los sentidos, y que conocen solo el mero fenómeno de las cosas, como pensaban Hume y Kant. El logos tiene que ver desde el principio con lo real; pero con lo que algo, ya real, es en realidad.

Reparemos en una obviedad consignada en la tesis enunciada: hay una distancia en la intelección entre el carácter meramente real de la cosa, actualizada intelectivamente en la aprehensión primordial de realidad, y lo que esa cosa es en realidad, tarea que le toca ya no a la aprehensión primordial sino al logos. Aún a riesgo de redundar, reparemos en una obviedad anterior a esta: en la intelección hay formalmente un momento preliminar al logos, la aprehensión primordial de realidad, motivo por el cual el logos en tanto intelección es un momento fundado y no fundante. Este acercamiento al logos como distanciación intelectiva da por supuesto ese momento preliminar de la mera actualización de lo real en la intelección².

En segundo lugar, debe decirse que esa intelección que es el logos, y que intelige lo que algo es en realidad, es un movimiento en dos fases: en su fase naciente, el logos se mueve de lo real hacia su campo de realidad, mientras que en su fase de plenitud torna desde el campo a lo real, dicho lacónicamente.

Primeramente, es la fase del movimiento de impelencia de la cosa real a un campo, al campo de "la" realidad. La impelencia es distanciación de lo que la cosa es en realidad. Y en efecto, para inteligir lo que una cosa entre otras es en realidad, lo primero que ha de hacerse

$1[\ldots]$ la palabra logos es un neologismo introducido por Zubiri con la función de común denominador de todos los actos de intelección dual. Por razón de su extensión, el término logos abarca las tres simples aprehensiones duales (perceptos, fictos, conceptos) y todas las formas y modos posibles de juicios. Por razón de su comprensión, el logos designa los actos de la primera intelección modal, mediante la cual alcanzamos descriptivamente la verdad dual de las cosas, tal como son aprehendidas unas desde otras en un determinado campo o mundo sentido". (Marquínez, 2013, p. 116)

2 A este problema está dedicado el primer volumen que trata el estudio de la inteligencia sentiente (cf. Zubiri, 1998). 
es "pararse a considerar" la cosa. Y pararse a considerar es ante todo una especie de suspensión intelectiva, es un tomar distancia de la cosa pero en ella y desde ella misma. (Zubiri, 2002, pp. 79-80)

El logos es movimiento, tanto en la primera fase, la de impelencia intelectiva, como en la que le sigue, la de intención intelectiva; pero es un movimiento que, instalado en la cosa real, sin abandonarla, se yergue sobre ella - pero desde ella - ; es un movimiento que debe entenderse como emanado desde el interior de la aprehensión primordial de realidad, cuyo trabajo es empujar desde el interior la compacción de la mera formalidad de realidad para que vaya lentamente abriéndose y ofreciendo su interior. En sus inicios más primigenios, esta fase ve aparecer los incipientes momentos de ese despliegue intelectivo que es el logos, despliegue ausente en la aprehensión primordial.

Una segunda fase en el movimiento del logos viene a completar el despliegue iniciado, en un movimiento en cierto sentido inverso al de la impelencia, pues ahora el logos se mueve del campo ${ }^{3}$ hacia la cosa real:

En esa distanciación la cosa real nos retiene tensos en ella, y por consiguiente revertidos a ella en un intentum por la tensión misma de la distancia. Es un movimiento del intentum para inteligir desde el campo lo que la cosa es en realidad. Por tanto es un referirnos desde el campo a la cosa: es intención intelectiva. El intentum se ha tornado en intención. Toma de distancia e intención intelectiva he aquí los dos momentos del movimiento intelectivo. (Zubiri, 2002, p. 80)

En la intelección que es el logos, este empieza por erguirse sobre la realidad y desde ella, como si se quisiera separar de ella; pero no lo puede hacer, porque no hay logos puro sino logos habitado de realidad, logos sentiente ${ }^{4}$. Este querer separarse de lo real - sin poder hacerlo jamás - trae de regreso al logos hacia lo real - de donde nunca se ha ido-; pero, dado que no es ya el logos que tira hacia fuera sino el logos que viene del intento de alejarse, se puede decir que ya no es el mismo del momento del erguirse, porque ahora está enriquecido por lo que ha visto y traído desde el campo de realidad, tal como le ha sucedido al que, habiéndose liberado y salido de la caverna, regresa después a ella (cf. Platón, República, 514a y ss.): ese enriquecimiento queda condensado en su estar tenso de regreso en relación con aquello sobre lo que se mantiene erguido.

De manera condensada - y muy compacta - han quedado enunciadas en los dos momentos anteriores las ideas que ciñen con rigor lo que Zubiri entiende

3 'El concepto de 'campo' es uno de los más importantes de la filosofía de Zubiri y uno de los términos técnicos de su obra que parecen más felices; se trata, además, de uno de los aspectos que mejor retratan el talante de un pensamiento inclinado siempre a un enfoque estructural de las cosas, en el cual las prioridades del sistema dominan siempre sobre los elementos aislados". (Pintor-Ramos, 1994, p. 131)

4 "El logos es sentiente porque intelige la realidad como la formalidad de ciertos contenidos sentidos. Claro que hay realidades postuladas y construidas, como en las matemáticas y la ficción, pero tal postulación constructiva se apoya en la misma realidad de las cosas que son reales en y por sí mismas. A la vez, el logo es sentiente porque es una intelección que inexorablemente recorre la realidad según la cual quedan los contenidos sentidos: un recorrido en la realidad desde su aprehensión compacta hasta su aprehensión diferencial, dual”. (Solari, 2008, p. 836) 
por logos. Las páginas siguientes son una profundización comprensiva - encaminada a mostrar su radicalidad respecto a todo otro modo del logos - solo en la primera fase del logos, la fase de la distanciación, la fase del erguirse de la intelección como logos, desde la aprehensión primordial de realidad.

Sin embargo, no podemos ir directamente a esa impelencia intelectiva que es el logos como distanciación en su primera fase, sino que, para mayor comprensión del asunto y sus deslindes con otros conceptos de logos, tal como el de Heidegger en el §7 de Ser y Tiempo (Heidegger, 2003, pp. 50-62), tenemos que empezar con unas ideas que preceden al tema, y al mismo tiempo debemos hacer algunos rodeos en torno al problema de la intelección.

\section{Antecedentes}

La primera de las ideas antecedentes al logos como distanciación es la tesis central de la noología de Zubiri, expuesta en su trilogía sobre la inteligencia sentiente, especialmente en el primer volumen titulado Inteligencia Sentiente. *Inteligencia y Realidad (1998). Esa tesis sostiene que el hombre es una inteligencia sentiente $\mathrm{e}^{5}$, no un animal racional, un ente que siente y razona; ni tampoco es el hombre el ente al que en su ser le va su mismo ser y el ser de los demás entes, es decir, Dasein (cf. Heidegger, 2003, p. 36). ¿Y cuál es la diferencia entre las tres expresiones, si al menos en dos de ellas se da el caso de que tenemos un concepto que se refiere a la sensibilidad y otro que nombra a la razón o inteligencia? No son solo tres expresiones diferentes, sino tres comprensiones distintas de la realidad de la intelección. La diferencia es que en la perspectiva que hace del hombre un animal racional - de Parménides a Husserl - hay una contraposición entre el sentir y el razonar del hombre ${ }^{6}$. Mientras que el sentir realiza su propio acto completo, que consiste en recibir las impresiones que le vienen del medio para ponerlas a disposición de la razón, esta a su vez realiza el suyo, que consiste en abstraer y formalizar en conceptos e ideas el material proporcionado por los sentidos. El ejemplo canónico del trabajo que por separado lleva a cabo cada facultad es Kant:

Si llamamos sensibilidad a la receptividad que nuestro psiquismo posee, siempre que sea afectado de alguna manera, en orden a recibir representaciones, llamaremos entendimiento a la capacidad de producirlas por sí mismo, es decir, a la espontaneidad del conocimiento. Nuestra naturaleza conlleva el que la intuición sólo pueda ser sensible, es decir, que no contenga sino el modo según

5 Dice Germán Marquínez que "Zubiri fue el primero en oponerse a este secular dualismo, afirmando la radical unidad entre el sentir e inteligir humanos, dos formalidades de un mismo acto, ejecutado por una única voluntad llamada inteligencia sentiente". (2013, p. 112)

6 Sorprende que en el propio Husserl encontremos expresiones tan marcadas sobre esta dicotomía: "Distinguimos aquí sensibilidad y razón. En la sensibilidad distinguimos la protosensibilidad, que no tiene nada de sedimentos de la razón, y la sensibilidad secundaria, que nace de una producción de la razón. En conformidad con ello dividimos también la razón en razón primigenia, intellectus agens, y razón sumergida en sensibilidad". (Husserl,1997, p. 386). 
el cual somos afectados por los objetos. La capacidad de pensar el objeto de la intuición es, en cambio, el entendimiento. Ninguna de estas propiedades es preferible a la otra: sin sensibilidad ningún objeto nos sería dado y sin entendimiento, ninguno sería pensado. Los pensamientos sin contenido son vacíos; las intuiciones sin conceptos son ciegas. (Kant, 2002, p. 93 [B 75] ${ }^{7}$

Explicar en qué consiste la continuidad entre lo sensible y lo racional en el hombre, cuáles son los términos en los que se da, cómo se pasa de lo sensible a lo racional, cómo es que los seres humanos sentimos cosas particulares pero pensamos conceptos universales, ha sido una de las cruces de la filosofía, porque envuelve la paradoja entera del ser humano en tanto que es una cosa del mundo, como cualquier otra, por un lado, pero al mismo tiempo es el que lo intelige y significa, por el otro ${ }^{8}$.

Pero la oposición entre el sentir y el inteligir, acciones propias del ser humano, se desvanece cuando se muestra con toda evidencia que, en el caso de esa realidad humana que soy cada vez yo, el sentir humano necesita formal y exigitivamente al inteligir para llevar a cabo su acto completo, lo mismo que el inteligir precisa del sentir para realizar su acto completo. Partir del inadvertido prejuicio de que el sentir de los animales y el de los hombres ha de ser formalmente igual, así como partir, del otro lado, del prejuicio de que el logos de Dios y el logos del hombre ha de ser al menos análogo, ha impedido ver en qué consisten el inteligir y el sentir en el hombre mismo, tal como lo vive y se da en la realidad humana, distribuido en todas las personas. Si hacemos epojé de lo que de común haya entre el sentir animal y el sentir humano, lo mismo que de lo que de común haya entre el logos divino y el humano, ¿qué nos queda? ¿qué pasa al primer plano? O preguntado de manera directa: ¿qué es inteligir y que es sentir en el hombre? La respuesta de Zubiri a estas preguntas abre un ámbito novedoso pero, en el corazón mismo de la tradición, de evidencia contundente: "Inteligir consiste formalmente en aprehender lo real como real, y [...] sentir es aprehender lo real en impresión" (Zubiri, 1998, p. 12). Ambos, el sentir y el inteligir humanos, están volcados desde el principio directamente a lo real: el hombre siente lo real e intelige lo real. No siente únicamente unas impresiones que son solo suyas, afecciones de la propia subjetividad, sino que siente que eso que le afecta es real, que es de suyo algo diferente a la mera afección; no intelige datos que formaliza o abstrae para obtener ideas, sino que intelige la realidad, algo que es en propio antes de ser inteligido. Dado que en la sensibilidad humana tenemos el caso de una potencia que precisa de otra, la inteligencia, para

7 Más que Husserl, parece ser Kant el principal interlocutor de Zubiri:

A primera vista, parece que las tres partes de Inteligencia Sentiente (1980-1983) se corresponden con la estructura de la primera de las Críticas kantianas [...]. Ahora bien, de la misma manera que ambos filósofos se mueven en planos diferentes e inconmensurables, se puede afirmar que Zubiri es el reverso de Kant y que, ciertamente, Inteligencia $y$ Realidad sí es el envés de la Estética trascendental, Inteligencia y Logos es el contrapunto de la Analítica trascendental y, finalmente, Inteligencia y Razón lo es de la Dialéctica trascendental. (Luengo, 1998, pp. 213-214)

8 El problema del conocimiento parece condensar la paradoja de la constitución del ser humano, tal como Husserl la describe: "La intersubjetividad universal, en la que se resuelve toda objetividad, todo lo que existe, no puede, manifiestamente, ser otra que la humanidad que innegablemente es una parte componente del mundo" (Husserl, 2008, p. 220). 
poder llevar a cabo su acto más propio - y lo mismo sucede con la inteligencia respecto a la sensibilidad - , repárese en que no se trata de dos actos, sentir e inteligir, sino de uno solo, el de la inteligencia sentiente; y que lo que ese acto hace es aprehender la realidad en impresión. Por este motivo, la inteligencia es sentiente, y no solo sensible; y la sensibilidad humana es ya intelección de lo real.

Ahora bien, en la estela de la inteligencia sentiente, ¿qué debe entenderse por realidad? La importancia de la aclaración de este punto es superlativa, porque el concepto de realidad está lastrado de muchos equívocos.

Aquí [en el tratado sobre la intelección] real significa que los caracteres que lo aprehendido tiene en la aprehensión misma los tiene "en propio", "de suyo", y no sólo en función, por ejemplo, de una respuesta vital. No se trata de la cosa real en la acepción de cosa allende la aprehensión, sino de lo aprehendido mismo en la aprehensión pero en cuanto está aprehendido como algo que es "en propio". Es lo que llamo formalidad de realidad. (Zubiri, 1998, p. 12)

La filosofía primera trata de lo que es primero en el orden de la realidad, en el orden de la inteligencia sentiente y en el orden de los primeros principios. La filosofía primera que trata sobre lo real en tanto real es metafísica; y la filosofía primera que trata sobre lo real en tanto haciéndose presente en la intelección es noología (cf. Villa-Sánchez, 2014). Nótese que ambas tratan sobre lo real, no sobre afecciones, ni sobre ideas, ni sobre el ser. Pues bien, desde la perspectiva de la noología, es realidad todo aquello que se hace presente en la intelección como siendo en propio eso que se hace presente, como siendo de suyo algo diferente a la intelección misma. La filosofía moderna inventó una serie de filtros (empiristas, racionalistas e idealistas) que, en el proceso del conocimiento, lo que hacían era poner a la razón cada vez más lejos de lo que había de ser conocido. Pero es exactamente al revés, pues lo real mismo es lo que se hace presente en la inteligencia, en el modo de formalidad de realidad. En el marco de la noología, en el que lo real en la intelección es formalidad de realidad, Zubiri suelta una frase que puede llevar a confusión si se la transpone al terreno de la metafísica. Dice que "es imposible una prioridad intrínseca del saber sobre la realidad ni de la realidad sobre el saber. El saber y la realidad son en su misma raíz estricta y rigurosamente congéneres. No hay prioridad de lo uno sobre lo otro" (Zubiri, 1998, p. 10). Lo real como tal es anterior a toda intelección; y Zubiri dedicó al asunto ese tratado metafísico mayor titulado Sobre la Esencia. Pero lo real como formalidad de realidad en la intelección es cooriginario con esa intelección y, en este caso, se los debe tratar bajo esta condición. Es este un asunto decisivo en el corazón mismo de la noología, plano en el que nos vamos a mover al tratar del logos como distanciación intelectiva.

La segunda idea que hay que poner como antecedente en el acercamiento al logos como distanciación intelectiva deriva de manera directa de la afirmación de que el hombre es una inteligencia sentiente, y que en la intelección realiza un solo acto $-\mathrm{y}$ no dos - que consiste en aprehender lo real, y no solo impresiones o fenómenos. Pues bien, ahora ha de preguntarse por la índole formal del acto 
que realiza dicha inteligencia sentiente. Aunque ya hablamos del asunto, es necesario en este momento decirlo explícita y enfáticamente: el acto formal de la intelección es la mera actualidad de lo real, el hacerse presente lo real en la intelección como siendo de suyo eso que se hace presente. Esta afirmación toma las cosas del estado en el que las dejó el giro criticista de Kant, y da marcha atrás $180^{\circ}$. ¿Hay que desandar solo la mitad del camino? Sí. Porque Kant tenía razón; pero no la tenía toda. Por lo tanto, no se puede volver al mismo punto en que estaba la teoría del conocimiento antes de 1781, por los innegables progresos que supone la Crítica de la Razón Pura. El acto radical de la inteligencia sentiente es modestísimo: la mera actualidad de lo real, el mero hacerse presente lo real en la intelección como algo que es en propio, con anterioridad a su hacerse presente. A explicar este complicado asunto dedica Zubiri Inteligencia Sentiente. *Inteligencia y Realidad (1998).

Debemos invocar una tercera idea antecedente. Porque si bien la intelección es, radicalmente, mera actualización de lo que lo real es de suyo como formalidad de realidad, la intelección no es solo esta primordial actualización. ¿Por qué? Porque habiéndose hecho presente lo real intelectivamente, la inteligencia quiere saber lo que lo real es en realidad. Esto fuerza de manera intrínseca el despliegue de la intelección: de la realidad meramente presente, hacia lo que la realidad es en realidad. La intelección no sale de la realidad, sino que se despliega hacia lo que dicha realidad es en realidad. El momento del "en realidad" de la realidad hecha presente en la intelección no es un momento posterior, añadido, sino que en la aprehensión primordial de realidad se hace presente una cosa como estando entre otras cosas, como perteneciendo a un campo de realidad ${ }^{9}$.

La relación entre logos y campo de realidad ${ }^{10}$, como primer despliegue de la actualidad de lo real en la intelección, es el tema de la primera de las tres secciones que conforman Inteligencia y Logos, el segundo volumen que estudia la inteligencia sentiente. La modestia del primer y radical momento que es la mera actualización de lo real en la intelección viene calificada por el hecho de que en ese momento la intelección no dice nada sobre lo real: solo lo hace presente como algo compacto, total, completo; distinto de la intelección, pero en ella. Al decir algo sobre lo real se ve impelida la intelección - ¡como sacudida desde dentro! - , porque cada cosa real se hace presente estando en función de otras cosas reales: esa es precisamente la tarea del logos.

9 "El campo no es algo que se añada a la realidad individual de una cosa, sino que se trata de dos dimensiones en distinta dirección que conforman su realidad de modo unitario; de este modo, una cosa es real en unos determinados contenidos concretos, los cuales, a su vez, configuran un campo de realidad en el que quedan dados como reales. El campo es una dimensión de las notas mismas que aparece como algo físico; al mismo tiempo, las notas son reales en un campo, que es un aspecto de su formalidad de real. Por tanto, no ha de entenderse el campo como si fuese resultado de alguna actividad del logos, pues se trata de algo que está dado en toda aprehensión primordial de realidad...". (Pintor-Ramos, 1994, p. 133)

10 "El hecho de que nunca captemos una sola cosa, un solo contenido, un solo contenido sino varios, pone de manifiesto el carácter campal de nuestra aprehensión. No se trata simplemente de una variedad, sino de una variedad unificada. Es el segundo momento básico del campo: su carácter de totalidad. En él se pone de relieve su estructura unitaria: primer plano, centro, fondo, periferia, horizonte, panorama, etc.". (Bañón, 1999, p. 86) 
Estas tres ideas - que el hombre es inteligencia sentiente, que la intelección es mera actualización de lo real, y que el logos es actualización de lo que lo real es en realidad- nos dejan ahora en condiciones de exponer qué es el logos ${ }^{11}$ como distanciación intelectiva. Ahora bien, en la distanciación intelectiva nos encontramos con dos momentos de especial importancia: la retracción intelectiva y la simple aprehensión. ¿Qué debe entenderse por cada uno de ellos en esa estructura formal ${ }^{12}$, no procesual, que es la inteligencia sentiente, específicamente el logos sentiente?

\section{Retracción intelectiva}

Tenemos que hacer un rodeo conceptual para llegar a ese momento que es la retracción intelectiva del logos. Lo real, tanto en propio como en la intelección, tiene un momento individual y un momento campal, que conforman la estructura del desdoblamiento de lo real. Ese doble momento es el que queda explicitado en el acto que intelige lo que lo real es en realidad, pues en la distancia que hay entre el momento individual y el momento campal es precisamente en la que la aprehensión primordial de realidad deviene logos por la exigencia misma de la distancia, o mejor dicho, de la distanciación entre el momento primero y el segundo. La unidad de lo real en la intelección desdoblada, en su momento campal, es lo que constituye la distancia intelectiva. Y este es precisamente el momento en el que debemos evitar a toda costa que el concepto de espacio físico se filtre en la comprensión correcta de la distancia intelectiva: "la distancia es un momento intrínseco a la cosa, es algo en ella misma” (Zubiri, 2002, p. 83). ¿Qué significa que la distancia es un momento interior a lo real individual-campal?

En esta distanciación sus dos momentos no están correlativamente distanciados. Lo que la cosa real individual es en realidad queda distanciado de esta realidad como realidad individual. Es decir, se mantiene la realidad de la cosa individual tanto en su formalidad de realidad como en su contenido, pero nos distanciamos por lo que concierne a lo que es "en realidad", es decir, hacemos del campo algo autónomo, un campo que ha de recorrerse. En esta distanciación queda instalada la cosa real individual en el campo de realidad. Por tanto, no salimos ni fuera de la cosa real misma ni fuera del campo de realidad, sino que nos quedamos en su momento campal para inteligir desde él lo que es en realidad su momento individual mismo. Vamos entonces en la cosa real desde su momento campal hacia su momento individual, la inteligimos campalmente. Esto es, recorremos la distancia como momento

11 [...] para Zubiri, el logos no es algo distinto de la inteligencia, sino un modo ulterior de la intelección sentiente, que tiene dos momentos: en el primero, el logos elabora perceptos, fictos y conceptos acerca de lo que las cosas previamente aprehendidas 'serían' dentro de un determinado medio o campo". (Marquínez, 20013, p. 115).

12 La tesis de Espinoza (2000) es que una realidad que es de suyo estructura, sustantiva, y no sustancia requiere un logos constructo. 
interno de la cosa, recorremos la dualidad como unidad en desdoblamiento. (Zubiri, 2002, p. 83)

Lo real se hace presente en la intelección de manera compacta y de manera desdoblada: como meramente real, como algo que es en propio lo que se hace presente, aunque no podamos saber que es tal o cual cosa. La intelección se mueve entre la realidad innegable de aquello que se hace primordialmente presente y lo que aquello sea "en realidad", una vez que lleguen la evidencia y la verdad - aunque estos son dos momentos que están todavía muy lejos en la trayectoria de esta exposición-. No sabemos lo que aquello que se ve a lo lejos es en realidad; no sabemos cuál de todos los contenidos llenará lo que ahora es mera forma de realidad. Pero ya sabemos con absoluta certeza que eso es real, que es algo en propio. Para descartar las posibilidades, para decantarse por una opción, la intelección en cuanto logos ha de ir y venir entre el momento individual de lo real y ese momento de desdoblamiento campal, en el que por ahora está detenido esperando decantarse por una de las opciones. Este "planear" sobre las opciones, sin poder aún aterrizar en alguna de ellas, tiene su propio momento de autonomía, su propia y relativa independencia, que con toda propiedad debe ser llamado campo intelectivo de la realidad.

Se toma distancia intelectiva de la cosa real en el interior mismo de la cosa real, sin decantarse todavía por alguna de las opciones de lo que eso es en realidad. ¿Cómo podría llamársele con más precisión y propiedad a este momento del logos en el que se va de lo meramente real, pero se frena, se detiene, planea, se contiene, sobre las diversas opciones que se le ofrecen para completarse como intelección? Zubiri lo llama retracción intelectiva: “es intelección de la cosa real dejando en suspenso lo que es en realidad... Estar lanzados por la cosa formalmente real al campo de 'la' realidad es dejar en suspenso retractivamente lo que la cosa es en realidad" (Zubiri, 2002, p. 84). La intelección se vuelve logos en esa reactualización que pretende saber lo que algo es en realidad; pero en ese movimiento hace el esfuerzo por contenerse, por no precipitarse, por mantener en suspenso momentáneamente su última palabra sobre lo que aquello sea (cf. Zubiri, 2002).

El momento de la retracción intelectiva del logos es un momento de la estructura formal de la intelección; por este motivo, el ejemplo de la incertidumbre que nos embarga, en relación a si lo que se ve a lo lejos es en realidad un pequeño arbusto o es otra cosa, puede llevar a pensar que en la retracción se trata del momento de un proceso empírico, que sucedería algunas veces, pero otras no. Sin embargo, dado que se trata de un momento formal y no empírico, siempre que hay intelección campal hay esa suspensión retractiva del logos planeando, pausándose provisionalmente, conteniéndose, sobre lo que lo real es en realidad. Aunque la intelección y la realidad son dos cosas diferentes tienen, sin embargo, un origen común en la intelección, de modo que en ese momento de la cooriginación hay una especie de unidad compacta entre inteligencia y formalidad de realidad. En ese momento, la inteligencia no está ni por abajo ni por arriba de la realidad sino que, dado que comparten el origen, están exactamente en el mismo plano. 
Pero lo real en la intelección se desdobla, y exige ser reactualizado en una intelección que es también forzada a desdoblarse de mera aprehensión primordial en logos. De camino hacia lo que lo real es en realidad, el logos se encuentra en una retracción que le retiene provisionalmente de tomar una de las posibilidades que le ofrece la misma realidad. La retracción intelectiva mantiene tenso al logos entre la mera realidad y su campo, entre la mera realidad y el "en realidad" de la cosa: el logos jamás se olvida de lo real, aunque en este primer momento se encamine hacia el campo como de espaldas a lo real. Más bien sucede que "en la retracción inteligimos 'la' realidad como algo abierto a lo que las cosas pueden ser en ella. Por tanto, estar en esta forma en 'la' realidad, es estar liberado por lo pronto de lo que las cosas son en realidad" (Zubiri, 2002, p. 85). De expresiones como estas, sembradas en los momentos más agónicos de la reflexión, nace la tesis de que la filosofía de la realidad tiene en su horizonte una crítica frontal a la tesis de la conciencia intencional de Husserl y a la ontología fundamental de Heidegger. Mientras este adscribe al Dasein el existenciario del estado de abierto y la estructura del ser-en-el-mundo, estructura en la que el ser del ente queda "libre para", Zubiri demuestra que la realidad es de suyo abierta y da de sí (cf. Zubiri, 1995), sea inteligida o no lo sea, pues hacerse presente en la intelección es solo una de las vías que lo real en tanto abierto puede recorrer. Y en tanto abierta, la realidad queda liberada para hacer por un abanico de posibilidades amplísimo en el dar de sí. Ni el estado de abierto de lo real, ni su libertad para dar de sí de infinitas maneras, penden en modo alguno de la intelección. Según la idea de la cita, en la retracción intelectiva, en ese compás de espera que abre el logos antes de su decantación por alguna de las alternativas, se alumbra con especial brillantez el estado de abierto de lo real actualizado en aprehensión primordial, pues se hace presente como pudiendo ser eso, esto o aquello. Pero en este estado de abierto de la realidad se muestra también el estado de libertad en que queda respecto a ser llenada por un contenido u otro.

La función del logos es hacerse cargo de lo que lo real es en realidad; pero en el camino que le lleva a cumplir con dicha función se encuentra con esa retracción que le suspende provisionalmente la primera fase del camino, y lo mantiene y sostiene planeando sobre diversas posibilidades de lo que sería ese "en realidad" (cf. Zubiri 2002, pp. 85-86). Este logos tenso, a medio camino entre lo real y su campo, entretenido en lo que lo real sería, deviene en lo que ahora debe ser llamado con toda propiedad simple aprehensión. La retracción intelectiva del logos es la que da origen a la simple aprehensión.

\section{La simple aprehensión}

¿Qué significa esta vieja expresión? ¿Conserva acaso solo su sentido escolástico? $¿ \mathrm{O}$ con ella se quiere decir algo nuevo, conservando, sin embargo, algo de su antiguo sentido? Ya se puede adivinar que hay algo nuevo en lo antiguo. "En impelencia, la intelección ya no es aprehensión primordial de realidad, sino simple aprehensión, mero término de intelección. Lo que la cosa es 'en realidad' es ahora, 
y por lo pronto, simple aprehensión. Simple significa ahora ser mero término de aprehensión" (Zubiri, 2002, p. 86). En la aprehensión primordial de realidad resuena esa idea clásica de la simple aprehensión. En la intelección se trata efectivamente de aprehensión. Pero no de lo que de inteligible hay en la cosa particular, y que es captado por el logos en su inicial quehacer como algo simple, lejos aún de la complejidad que lo inteligido en tanto tal alcanzará en el intelecto, porque anterior a esa simple aprehensión, a ese captar (cogitare) lo inteligible de lo particular, hay una aprehensión primordial que tiene como característica hacer presente lo real mismo, y no una mera especie sensible o inteligible. La simple aprehensión de la Escolástica - en el caso de Santo Tomás, por ejemplo (cf. Muñoz, 2012) - está conceptualizada desde la perspectiva que entiende a la sensibilidad y a la inteligencia como dos facultades contrapuestas, distintas, completas, y realizando cada cual su acto propio separadamente. Pero las cosas cambian cuando la sensibilidad humana y la intelección se reclaman una a la otra para completarse, y terminan ejecutando un solo acto y no dos. Ahí la simple aprehensión, como primera captación de lo inteligible de algo particular, pierde ese lugar en razón de que la inteligencia va de la realidad de algo en la intelección a lo que eso es en realidad. El lugar de la simple aprehensión está ahora en el trayecto de lo real a lo que algo es en realidad: en esa retracción que sufre el logos antes de posarse sobre una de las alternativas del "en realidad" que libera lo real. En la idea clásica de simple aprehensión, en este momento del acto intelectivo se prescinde del contenido de la cosa para quedarse solo con aquello que es inteligible en ella; "pero la retracción conserva todo el contenido de la cosa como realidad, y lo que deja en suspenso no es 'la realidad', sino lo que la cosa es 'en realidad'. Continúa la realidad siendo el 'de suyo', pero no sabemos qué es en realidad este 'de suyo'" (Zubiri, 2002, pp. 89). La simple aprehensión se mueve ya en lo real, en ese momento en el que la intelección quiere saber qué es en realidad eso que se le ha hecho presente. Y no se trata, dice Zubiri, de una mera sutileza (cf. Zubiri, 2002).

La simple aprehensión es un principio de inteligibilidad de lo que una cosa real es en realidad, no el primer despojamiento de los caracteres particulares de la cosa como condición para ser inteligida. La inteligencia no despoja a la cosa que se actualiza de nada, no le quita sus propiedades, sus contenidos, para quedarse solo con su especie. En el ir de lo aprehendido como real a lo que la cosa es en realidad, la intelección va deviniendo logos desde la aprehensión; y ese logos, en su ir hacia el campo de lo real, sufre una especie de retención que lo hace sobrevolar las diversas opciones que se le ofrecen para completar su trayectoria, para ser logos pleno. En esta retención el logos queda suspendido de manera tensa entre lo meramente real y lo real campal, quedando ambos momentos como principio de inteligibilidad de lo que la cosa es en realidad. Este "quedar tenso" entre ambos momentos es entonces ese principio de inteligibilidad que es la simple aprehensión. Dado que el momento campal no le llega a lo real individual después, sino que formalmente es un momento de su propia constitución y de su propia actualización, ese principio de inteligibilidad tampoco le llega a lo inteligido, ni al acto de intelección, como desde fuera, sino que es también un momento formal de la cooriginariedad de realidad e inteligencia. 
En este momento de transición hacia lo que las cosas son en realidad, la simple aprehensión se alza como un necesario principio de inteligibilidad del campo de lo real, es decir, como un principio de inteligibilidad necesario para poder saber lo que unas cosas son entre otras, y para poder saber la funcionalidad de unas cosas respecto de otras. Ambos momentos, el del "entre" y el de la "funcionalidad", son constitutivos del momento campal de lo real. El logos lleva en su seno, como momento estructural suyo, esa especie de soplo que es la simple aprehensión como principio de inteligibilidad de lo que las cosas son en realidad.

¿En qué condición queda lo real aprehendido en ese momento estructural del logos que es la simple aprehensión? Lo real y el "en realidad" de la cosa quedan provisionalmente como mero principio de inteligibilidad. Pero ¿qué significa esto más precisamente? "Ser mero término es haber quedado suspendido el contenido de la realidad en tanto que contenido, con lo cual este contenido ya no es propiamente real sino irreal. En retracción, lo que las cosas son en realidad constituye por lo pronto el orbe de lo irreal" (Zubiri, 2002, p. 91). ¿Y qué debe entenderse por lo irreal? ¿Acaso es aquello que no existe? ¿O aquello que solo existe en la imaginación y en la inteligencia, pero no en la realidad extramental? Lo irreal debe entenderse en la línea de lo real como siendo en propio, y no en relación con la existencia ni con lo extramental. Un personaje literario, un sueño, un desvarío mental, una ilusión son tan reales, es decir, de suyo y algo en propio, como la planta que crece en el jardín o el perro que ladra en el campo.

El rodeo que la intelección de lo real debe dar en la simple aprehensión por la irrealidad mostrará hasta qué punto ese principio de inteligibilidad es más que una mera sutileza, y nos llevará hacia la necesidad de distinguir varios modos de la simple aprehensión (percepto, ficto y concepto), porque si bien la planta del jardín y un personaje literario, como Pedro Páramo de Juan Rulfo (Aureliano Buendía de García Márquez o El Quijote de Cervantes), son algo en propio, es claro también que su realidad tiene modos distintos. En la simple aprehensión lo real queda, pues, irrealizado y, por lo tanto, perteneciendo al ámbito de lo real. ¿Cómo es esto posible? Lo real se constituye en irreal en la simple aprehensión por "desrealización". "Y el 'des' no es un momento puramente negativo: si lo fuera la cosa no sería irreal sino arreal. Por tanto es un 'des' positivo: es la positiva inclusión en la realidad en forma de 'des'”' (Zubiri, 2002, p. 91). No es la realidad la que queda suspendida en su formalidad, porque tal cosa es imposible una vez que ha tenido lugar la actualización intelectiva; sino que lo que queda suspendido en ese momento del "des" de la desrealización es que lo formalmente real sea tal o cual contenido, tal o cual cosa en concreto. Ese momento de la desrealización por la que ha de pasar todo logos es el que abre el abanico infinito de las posibilidades para que el logos pueda decirse de varias maneras, por ejemplo, en el mito, en la literatura, en la ciencia, en la filosofía, en la teología, en el lenguaje de la cotidianidad media, etc., y le pone límites a la soberbia de la primacía de alguno de esos modos sobre los demás. Ya se deja ver cómo conecta con ese momento de la desrealización de lo real en el logos el 
estado de libertad ${ }^{13}$ en que queda la formalidad de realidad para realizarse: un mismo contenido puede ser desarrollado -ir del momento de desrealización a su realización - en un apretado tratado científico, o convertirse en el material para un relato novelado (cf. Zubiri, 2002).

Realizar de un modo o de otro el contenido, por lo pronto retenido en la desrealización, no es de ninguna manera un acto de arbitrariedad, sino que se inscribe en la dinámica y en la fuerza de realidad que la formalidad tiene desde el principio de su actualización: un científico no decide a medio camino de su investigación cambiar de rumbo y terminar su tratado en un cuento de terror, como tampoco el escritor hará de su novela un tratado académico de ciencias naturales o filosofía. De modo que lo real, actualizado en una formalidad libre y abierta desde el principio, se encuentra por lo pronto desrealizado, en espera de plenitud, ciertamente, pero ya en dirección de uno u otro contenido.

La trayectoria de impelencia del logos, esa que va de lo real a lo que lo real es en realidad, alcanza su plenitud cuando lo desrealizado deviene realización en alguno de los modos de la simple aprehensión. Porque no hay simple aprehensión sin más, como si fuera neutra, limpia de toda impureza, vacía de todo resabio sentiente. Cuando ha acontecido la simple aprehensión, lo ha hecho ya en el modo del percepto, del ficto o del concepto. Estos tres modos de la simple aprehensión nombran tres dimensiones diferentes de lo real y de su actualización intelectiva, que se recubren entre sí, con la preeminencia de uno sobre los otros según el caso, y que se abren desde la condición de libertad que caracteriza el momento de desrealización de lo real. Ya sea en un percepto, un ficto o un concepto, llega a su plenitud la primera fase del logos. Así que debemos decir qué es lo que caracteriza a cada uno, como dimensión propia de la cosa real.

\section{Los modos de la simple aprehensión}

¿Qué es un percepto, el primero de los modos de la simple aprehensión en que el logos llega a su plenitud?

Ante todo, lo primero que puede quedar desrealizado en la retracción liberadora, es el contenido de la cosa real entera. No es la cosa prescindiendo de "la" realidad, sino "la" realidad terminando libremente en esta cosa como aquello que ésta sería en realidad. En su virtud, queda actualizada la cosa real en una dimensión propia suya: ser "esto". Aquí "esto" no significa "este", sino lo que es el "este": es el este en cuanto "esto". Aprehender "esta" cosa es lo que constituye la aprehensión primordial de realidad, por ejemplo la percepción. Pues bien, el "esto" de "esta" cosa, desrealizado, es el "esto" ya no "en cuanto real" sino "en cuanto percibido"; es

13 "Es de gran importancia esta introducción por parte de Zubiri del concepto de 'libertad' dentro del proceso intelectivo. Sin ella, el proceso de distanciamiento y reversión que opera el logos sería imposible y sin ese distanciamiento no cabría atribuir una verdad concreta a los diversos contenidos que conforman nuestro mundo intelectivo". (Pintor-Ramos, 1994, p. 141) 
el "esto" de la cosa en cuanto mero término de percepción [...] Reducir el contenido de la cosa real a percepto: he ahí la primera forma de la simple aprehensión. (Zubiri, 2002, p. 97)

El percepto es la posibilidad de las otras simples aprehensiones. En el camino de subida hacia la cima de la intelección propia del logos, iniciada con la aprehensión primordial de realidad, bien puede entenderse el percepto como el movimiento que da el primer paso desde esa cima hacia abajo, pero por la cara de lo real en tanto perceptuado, no en tanto meramente actualizado y percibido. De percibida, de aprehendida como real, la cosa deviene perceptuada como realidad en este modo de la simple aprehensión. Aunque no lo diga aún el lenguaje, la cosa en tanto perceptuada es eso que con el lenguaje podemos decir con el más neutro de los deícticos. Respecto de la intelección en la que se hace presente, lo real queda distinguido de dicha intelección como una totalidad que es por lo pronto un mero "esto". En el "esto" la formalidad de realidad queda perceptuada por primera vez en tanto inteligida en el logos; la retención del logos, que planeaba sobre la cosa antes de precipitarse sobre ella para llenarse con uno u otro contenido, empieza liberándose de esa retención con un "esto" en tanto meramente perceptuado. En la simple aprehensión en la que lo real queda en el modo del "esto", el logos retenido encuentra el primordial canal de liberación. El contenido de algo, no su formalidad de realidad, queda reducido por lo pronto a un logos perceptual - aún no de ficción ni conceptual - , a un mero "esto" compacto, entero, monolítico y sin grietas.

La simple aprehensión de una cosa real como mero percepto en tanto solamente "esto" es libre y, seguidamente, libre creación. La libertad creativa está en el origen del movimiento que lleva al logos a decantarse por uno u otro contenido, “porque la cosa real ciertamente es 'esto', pero reducir el 'esto' a mero percepto es una rigurosa creación" (Zubiri, 2002, p. 98). Pues si la intelección se mueve en una dirección en lugar de hacerlo en otra, ese "esto" será diferente en cada caso.

Muchas cosas actualizadas en la intelección quedan en el logos solo en el modo del logos perceptual, solo en tanto meramente percibidas, perceptuadas; pero en muchos otros casos, la simple aprehensión se da en el modo de la ficción y del concepto, y entonces lo real actualizado en la inteligencia sentiente como formalidad de realidad deviene realidad en ficción y realidad en concepto.

¿Qué es lo real en ficción? La ficción es otro de los modos en los que lo real en la intelección, vía el logos, ve liberada de manera creativa, en una simple aprehensión con caracteres diferentes a la perceptual, la plenitud a la que se encamina todo logos en ese movimiento de impelencia que lo lleva de lo real a lo que la cosa es en realidad. ¿En qué consiste, más precisamente, la liberación de lo real en ese modo de la simple aprehensión, dada al interior del logos, que es la ficción? En la simple aprehensión, en la que lo real queda perceptualmente entero como "esto", el logos se está moviendo ya en la realidad de lo actualizado intelectivamente: ya tengo sabido en este momento que lo real es en realidad; pero aún no sé ni qué es ese "en realidad", ni cómo es. 
Todo "esto" es un sistema unitario de notas reales. Según este sistema unitario, la cosa no es un mero complejo de notas reales cualquiera, sino de estas notas sistematizadas en una cierta "manera"; de suerte que si lo estuvieran de otra ya no serían la misma cosa sino justamente otra. Es decir, la cosa real en su "esto", tiene además de sus notas, el "cómo" de su sistematización. Reducido a percepto el "esto", conserva el "cómo perceptual". Pues bien, puedo retraerme liberándome en el "esto" mismo de su propio "cómo". La simple aprehensión queda entonces en franquía para crear el cómo. Claro está, no me limito a crear el "cómo" dejando intactas las notas, sino que las notas oriundas de perceptos pueden entonces ser libremente creadas para hacer de ellas un nuevo "cómo". El término de este movimiento intelectivo creador del "cómo" es un "cómo" fingido: es un ficto, un fictum. La simple aprehensión de la cosa como un "cómo" ficto es la ficción. (Zubiri, 2002, pp. 98-99)

No hay, sin más, la realidad perceptual. El momento perceptual está recubierto o por el momento ficticio o por el momento conceptual. Pero en ninguno de los dos casos como si se tratara de una capa superpuesta sobre otra que permanece inalterada. En el momento perceptual, el qué y el cómo de lo real son dinámicos, se mantienen dando de sí. En la simple aprehensión que es la ficción, el cómo del "esto" perceptual es liberado de forma creativa, de modo que lo real viene a quedar en un modo fingido, el modo de la ficción. El arte en general, la literatura en particular, no fingen la realidad, sino que sucede exactamente lo contrario: lo real queda en el modo de la ficción, en el modo de lo fingido. En esta zona de cosas la idea de realidad como formalidad del "en propio" nos ofrece uno de sus ricos rendimientos. Efectivamente, en Cien años de soledad, García Márquez no finge la realidad, no la inventa simplemente de manera arbitraria, sino que la realidad queda en el modo de la ficción: el lector sabe que en una novela como esta se le ofrece un conocimiento sobre la realidad, solo que se da una realidad novelada. Y esta realidad en tanto novelada tiene la misma densidad ontológica que la que tiene la realidad en un tratado de química o en uno de historia. De tal manera es esto así que, por ejemplo, en El llano en llamas de Juan Rulfo - o en cualquier otra obra literaria o producto de la ficción - , un buen criterio sobre la calidad de la obra es que en lo ficticio, en lo figurado, en lo simulado, se ofrezca lo real, lo que las cosas son de suyo, en su justa medida. ¿Quién, leyendo Pedro Páramo, esa otra maravillosa obra de Rulfo, no ve aparecer ante los ojos de su imaginación la realidad del México rural posrevolucionario? La maestría de la pluma del novelista ha tomado el "esto" perceptual de la realidad para reconducir, de manera libre y creativa, esa realidad en dirección a la ficción. Los ejemplos nos ayudan para mostrar de manera contundente que, en la adjudicación del cómo del "esto", propia de la simple aprehensión que es la ficción, es el "esto" mismo el queda en el modo de la ficción y no solo el cómo de sus notas. La comprensión de la realidad de la Revolución Mexicana, o la comprensión de la realidad del siglo xx de Colombia, no han quedado incólumes después de la obra de Rulfo y García Márquez, porque las propias notas del "esto" han sido libremente creadas. 
El tercer modo de la simple aprehensión es el concepto. ¿Cuál es el modo que tiene el concepto de recubrir un percepto y al propio ficto?

\begin{abstract}
Desrealizada la cosa por libre retracción, su "qué" queda irrealizado y reducido a un mero "qué" en cuanto aprehendido: es justo lo que llamamos concepto. Concepto no es algo primariamente lógico sino algo real: es el "qué-concepto". El concepto envuelve formalmente y físicamente el momento de realidad. El concepto es "la" realidad física misma como si fuera este "qué": concebimos qué sería realmente la cosa, lo que esta sería en realidad.... El concepto es, pues, la realidad terminada en libre "qué". (Zubiri, 2002, p. 101)
\end{abstract}

El concepto debe inscribirse en el movimiento de apertura y libertad de la propia realidad. Pudiendo realizarse de varias maneras, el qué queda desrealizado por la libre retracción de la intelección en una determinada dirección. En un concepto, la realidad de una cosa queda reducida a un "qué" en tanto que aprehendido: el concepto actualiza lo real en la intelección como un "qué" libremente creado. ¿Significa acaso que un concepto es producto de la libre creación, tanto como lo es un ficto en el mundo de la ficción? La respuesta tiene que ser afirmativa. La diferencia es que en el ficto lo que queda libremente desrealizado en retracción es el cómo de la realidad, mientras que en el concepto lo desrealizado es el qué. Ahora bien, el "qué" de la realidad puede ser llevado a su "qué" en tanto que aprehendido de muchas maneras, de modo que sobre un "qué" real podemos tener varias propuestas conceptuales. Una propuesta metafísica sobre la realidad, una teoría física sobre la naturaleza, una investigación sobre la ética o la política no son más que maneras libres y creativas de desrealización conceptual del "qué".

Debemos reparar en que el concepto nos dice lo que lo real sería en realidad. En la intelección debe distinguirse el momento de la mera actualización de lo real como real, en la que lo real no hace más que hacerse presente en la intelección. Pero a la intelección no le basta con que lo real se le haga presente, sino que quiere saber lo que lo real es en realidad. Y saber lo que lo real es en realidad exige perceptos, fictos y conceptos. Con el concepto, la intelección hace por saber el qué - no el "esto", ni el cómo- de lo que lo real es en realidad. Es por eso que, en el concepto, tenemos una especie de propuesta de lo que lo real sería en realidad: en un entramado conceptual se postula que el "qué" de algo en tanto aprehendido en realidad podría ser tal. La postulación y la hipótesis habitan el concepto desde el principio: en ese concepto se propone que lo que algo es en realidad podría ser esto; pero no se cierran las puertas a que haya otras posibilidades conceptuales que digan con más propiedad lo que lo real sería en realidad.

Percepto, ficto y concepto son los tres modos de la simple aprehensión. Con ellos hemos llegado al momento final de esa primera fase del logos que es la impelencia. 


\section{Conclusión}

El logos tiene una estructura dinámica. El logos intelige lo que lo real es en realidad: es por tanto un movimiento que va de lo meramente real a lo que eso, que ya es real, es en realidad. Este movimiento que es el logos tiene dos fases: en la primera fase el logos es impelido de lo real hacia el campo de realidad; sin dejar lo real, sin abandonarlo, el logos se mueve de la realidad de una cosa hacia la realidad de otras cosas. Es el momento de distanciación intelectiva dentro de lo real. En este movimiento encontramos los momentos de la retracción intelectiva y de la simple aprehensión en sus modos de percepto, ficto y concepto. Pero para ser pleno, el logos sentiente, en su estructura dinámica, tiene una segunda fase, en la que encontramos el momento de la afirmación, y con el cual se completa la estructura dinámica del logos. La evidencia y la verdad, que no hemos visto aparecer en estos análisis, pertenecen a la estructura medial del logos y no a su estructura dinámica. De manera que nos queda pendiente el estudio de la afirmación, lo mismo que el de la evidencia y la verdad. Solo entonces tendremos un panorama completo de la estructura esencial del logos sentiente. Pero ya con estos avances va mostrándose que afirmar que el logos equivale, sin más explicaciones, a $\delta \eta \lambda o \tilde{v} v$, es decir, a "hacer patente aquello de lo que se habla en el decir" (cf. Heidegger, 2003), es una ingente simplificación del logos sentiente, de la inteligencia sentiente, y de la cooriginariedad entre inteligencia y realidad.

\section{Referencias}

Bañón, J. (1999). Metafísica y Noología en Zubiri. Salamanca: ups.

Espinoza, R. (2000). El logos nominal constructo en el pensamiento de Xavier Zubiri. The Xavier Zubiri Review, 3(2000/2001), 121-132.

Heidegger, M. (2003). Ser y Tiempo. Madrid: Trotta.

Husserl, E. (1997). Ideas relativas a una fenomenología pura y una filosofía fenomenológica. Libro Segundo: Investigaciones fenomenológicas sobre la constitución. México: unAm.

Husserl, E. (2008). La crisis de las ciencias europeas y la fenomenología trascendental. Buenos Aires: Prometeo.

Kant, I. (2002). Crítica de la Razón Pura. México: Alfaguara.

Luengo, J. (1998). Zubiri y Kant. Bilbao: Universidad de Deusto. 
Marquínez, G. (2013). “'Inteligencia sentiente’ de X. Zubiri: desde una perspectiva lingüística”. Cuadernos de Filosofía Latinoamericana, 39(109), 103-126. Dor: https://doi.org/10.15332/s0120-8462.2013.0109.07.

Muñoz, C. (2012). En torno a dos lecturas posibles sobre el conocimiento de las esencias en Tomás de Aquino. Tópicos, 43, 123-151. Recuperado de http:// www.scielo.org.mx/pdf/trf/n43/n43a5.pdf

Pintor-Ramos, A. (1994). Realidad y Verdad. Las bases de la filosofía de Zubiri. Salamanca: ups.

Pintor-Ramos, A. (2006). Nudos en la filosofía de Zubiri. Salamanca: ups.

Platón. (2003). Diálogos IV. República. Madrid: Gredos.

Solari, E. (2009). El logos del teísmo según Zubiri. Pensamiento, 65(246), 833880. Recuperado de https://revistas.comillas.edu/index.php/pensamiento/ article/view/4790/4616

Villa-Sánchez, J. A. (2014). La actualidad de lo real en Zubiri. Crítica a Husserl y Heidegger. México: Plaza y Valdés.

Zubiri, X. (1995). Estructura Dinámica de la Realidad. Madrid: Alianza-FXZ.

Zubiri, X. (1998). Inteligencia Sentiente. *Inteligencia y Realidad. Madrid: Alianza-FXZ.

Zubiri, X. (2002). Inteligencia y Logos. Madrid: Alianza-FXZ. 